\title{
ON THE STABILITY OF CONVEX-VALUED MAPPINGS AND THEIR RELATIVE BOUNDARY AND EXTREME POINTS SET MAPPINGS*
}

\author{
MIGUEL A. GOBERNA ${ }^{\dagger}$, MAXIM I. TODOROV $\ddagger$, AND VIRGINIA N. VERA DE SERIO $§$
}

\begin{abstract}
This paper deals with the transmission of the main stability properties (lower and upper semicontinuity in Berge sense, and closedness) from a given closed-convex-valued mapping to its corresponding relative boundary and extreme point set mappings, and vice versa. The domain of the mappings considered in this paper are locally metrizable spaces and the images range on Euclidean spaces. Important examples of the class of mappings considered in this paper are the feasible set mapping and the optimal set mapping of convex optimization problems, for which the space of parameters is the result of perturbing a given nominal problem.
\end{abstract}

Key words. stability theory, set-valued mappings, convex hull mappings, relative boundary mappings, extreme points set mappings

AMS subject classifications. 49K40, 28B20

DOI. $10.1137 / 050632476$

1. Introduction. The main objective of the paper is to analyze the relationships between important pairs of mappings, one of them being the convex hull of the other, which frequently arise in convex optimization (convex systems), where, as a consequence of measurement or roundoff errors, the nominal problem $y_{0}$ (system $y_{0}$ ) is usually replaced in practice by perturbed problems (systems, respectively) having the same structure. Let us denote by $Y$ the set of all possible perturbed problems (systems) equipped with a certain pseudometric measuring the size of the perturbations and let $\mathcal{F}: Y \rightrightarrows \mathbb{R}^{n}$ be the set-valued mapping associating with each $y \in Y$ its feasible set or its optimal set (its solution set, respectively). Under mild conditions, $\mathcal{F}(y)$ is the convex hull of its boundary set bd $\mathcal{F}(y)$, its relative boundary set $\operatorname{rbd} \mathcal{F}(y)$, and/or its extreme points set $\operatorname{extr} \mathcal{F}(y)$ for all $y \in Y$. We denote these mappings from $Y$ to $\mathbb{R}^{n}$ as bd $\mathcal{F}, \operatorname{rbd} \mathcal{F}$, and extr $\mathcal{F}$, which are called boundary mapping, relative boundary mapping, and extreme points set mapping of $\mathcal{F}$, respectively. The connections between the stability properties of $\mathcal{F}$, bd $\mathcal{F}$, and extr $\mathcal{F}$ have been already analyzed in the particular context of linear semi-infinite systems ([3] and [4], respectively), where $Y$ is equipped with the pseudometric of the uniform convergence.

Throughout this paper we consider given an arbitrary convex-valued mapping $\mathcal{F}$ : $Y \rightrightarrows \mathbb{R}^{n}$, where the domain $Y$ is a locally metrizable space (i.e., $Y$ is equipped with the topology induced by an extended distance on $Y, \delta$, taking values on $\mathbb{R}_{+} \cup\{+\infty\}$ ), and its boundary mapping, relative boundary mapping, and extreme points set mapping,

* Received by the editors May 26, 2005; accepted for publication (in revised form) November 2, 2005; published electronically May 3, 2006.

http://www.siam.org/journals/siopt/17-1/63247.html

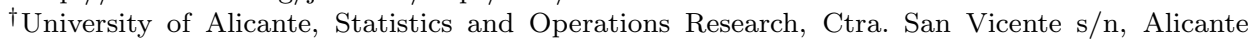
03071, Spain (mgoberna@ua.es). This author was supported by MCYT of Spain and FEDER of EU, grant BMF2002-04114-C02-01.

${ }^{\ddagger}$ Actuary and Mathematics, UDLA, 72820 San Andrès Cholula, Puebla, Mexico. On leave from IMI-BAS, Sofia, Bulgaria (maxim.todorov@udlap.mx). This author was supported by CONACyT of Mexico, grant 44003.

$\S$ Universidad Nacional de Cuyo, Faculty of Economics, Campus UNCUYO, Mendoza 5500, Argentina (vvera@fcemail.uncu.edu.ar). This author was supported by SECYT-UNCuyo of Argentina, grant 987/02-R-04. 
bd $\mathcal{F}, \operatorname{rbd} \mathcal{F}$, and extr $\mathcal{F}$. The relationships between $\mathcal{F}$ and bd $\mathcal{F}$, assuming that $\mathcal{F}=\operatorname{conv}$ bd $\mathcal{F}$, have been studied in [5]. In the same vein, this paper considers the relationships between the stability properties of $\mathcal{F}, \operatorname{rbd} \mathcal{F}$, and extr $\mathcal{F}$, assuming that $\mathcal{F}=\operatorname{conv} \operatorname{rbd} \mathcal{F}$ and $\mathcal{F}=$ conv extr $\mathcal{F}$, respectively. The finite dimension of the image space plays a crucial role in those arguments based on the compactness of the unit sphere or on Carathéodory's theorem.

Some of these relationships are direct consequences of basic results about arbitrary mappings $\mathcal{A}: Y \rightrightarrows \mathbb{R}^{n}$ and their corresponding convex hull mappings, conv $\mathcal{A}$ : $Y \rightrightarrows \mathbb{R}^{n}$, which associates to each $y \in Y$ the convex hull of $\mathcal{A}(y)$, i.e., $(\operatorname{conv} \mathcal{A})(y)=$ $\operatorname{conv} \mathcal{A}(y)$ for all $y \in Y$. Although some results on the transmission of stability properties between $\mathcal{A}$ and $\operatorname{conv} \mathcal{A}$ are already known (see, e.g., [6] and [1]), we provide proofs of other results which will be used in what follows. Thus, for each stability property, we start analyzing the relationships between $\mathcal{A}$ and conv $\mathcal{A}$, and then we exploit the properties of the images of $\mathcal{F}, \operatorname{rbd} \mathcal{F}$, and extr $\mathcal{F}$ in order to obtain the relationships between these mappings; section 3 deals with the lower semicontinuous (lsc) property and section 4 with the upper semicontinuous (usc) property and closedness.

Let us introduce some additional notation. Given $X \subset \mathbb{R}^{n}$, aff $X$ denotes the affine hull of $X$. From the topological side, bd $X, \operatorname{rbd} X, \operatorname{int} X, \operatorname{rint} X$, and $\operatorname{cl} X$ represent the boundary, the relative boundary, the interior, the relative interior, and the closure of $X$, respectively. If $X$ is convex, its set of extreme points is denoted by extr $X$. The Euclidean norm in $\mathbb{R}^{n}$ will be denoted by $\|$.$\| and the open ball centered$ at $x$ and radius $\varepsilon>0$ by $B(x ; \varepsilon)$. If $X$ is a convex set and $x \in X$, then

$$
B(x ; \varepsilon) \cap \operatorname{rbd} X=\emptyset \Longrightarrow B(x ; \varepsilon) \cap \operatorname{aff} X \subset \operatorname{rint} X
$$

for all $\varepsilon>0$.

The standard simplex in $\mathbb{R}^{n+1}$ is

$$
S:=\left\{\left(\lambda_{1}, \ldots, \lambda_{n+1}\right) \in \mathbb{R}_{+}^{n+1} \mid \sum_{i=1}^{n+1} \lambda_{i}=1\right\} .
$$

For the sake of completeness, we recall the stability concepts and some basic results for set-valued mappings that we shall consider in this paper. Let $\mathcal{M}: Y \rightrightarrows \mathbb{R}^{n}$ be a set-valued mapping with its domain $\operatorname{dom} \mathcal{M}:=\{y \in Y \mid \mathcal{M}(y) \neq \emptyset\}$. The following semicontinuity concepts are due to Bouligand and Kuratowski (see [1, section 1.4]).

We say that $\mathcal{M}$ is lower semicontinuous at $y_{0} \in Y$ in the Berge sense if, for each open set $W \subset \mathbb{R}^{n}$ such that $W \cap \mathcal{M}\left(y_{0}\right) \neq \emptyset$, there exists an open set $V \subset Y$, containing $y_{0}$, such that $W \cap \mathcal{M}(y) \neq \emptyset$ for each $y \in V$. Obviously, $\mathcal{M}$ is lsc at $y_{0} \notin \operatorname{dom} \mathcal{M}$ and $y_{0} \in \operatorname{int} \operatorname{dom} \mathcal{M}$ if $\mathcal{M}$ is lsc at $y_{0} \in \operatorname{dom} \mathcal{M}$.

$\mathcal{M}$ is upper semicontinuous at $y_{0} \in Y$ in the Berge sense if, for each open set $W \subset$ $\mathbb{R}^{n}$ such that $\mathcal{M}\left(y_{0}\right) \subset W$, there exists an open set $V \subset Y$, containing $y_{0}$, such that $\mathcal{M}(y) \subset W$ for each $y \in V$. If $\mathcal{M}$ is usc at $y_{0} \notin \operatorname{dom} \mathcal{M}$, then $y_{0} \in \operatorname{int}(Y \backslash \operatorname{dom} \mathcal{M})$.

If $\mathcal{M}$ is simultaneously lsc and usc at $y_{0}$ we say that $\mathcal{M}$ is continuous at this point.

$\mathcal{M}$ is closed at $y_{0} \in \operatorname{dom} \mathcal{M}$ if for all sequences $\left\{y_{r}\right\}_{r=1}^{\infty} \subset Y$ and $\left\{x_{r}\right\}_{r=1}^{\infty} \subset \mathbb{R}^{n}$ satisfying $x_{r} \in \mathcal{M}\left(y_{r}\right)$ for all $r \in \mathbb{N}, \lim _{r \rightarrow \infty} y_{r}=y_{0}$ and $\lim _{r \rightarrow \infty} x_{r}=x_{0}$ (in brief, $y_{r} \rightarrow y_{0}$ and $\left.x_{r} \rightarrow x_{0}\right)$ one has $x_{0} \in \mathcal{M}\left(y_{0}\right)$. If $\mathcal{M}$ is usc at $y_{0} \in \operatorname{dom} \mathcal{M}$ and $\mathcal{M}\left(y_{0}\right)$ is closed, then $\mathcal{M}$ is closed at $y_{0}$. Conversely, if $\mathcal{M}$ is closed and locally bounded at 
$y_{0} \in \operatorname{dom} \mathcal{M}$ (i.e., if there is a neighborhood of $y_{0}$, say $V$, and a bounded set $A \subset \mathbb{R}^{n}$ containing $\mathcal{M}(y)$ for every $y \in V)$, then $\mathcal{M}$ is usc at $y_{0}$.

Finally, $\mathcal{M}$ is lsc (usc, closed, locally bounded) if it is lsc (usc, closed, locally bounded) at $y$ for all $y \in Y$.

Without entering in details we would like to mention that there are other notions of lower and upper semicontinuity as lsc and usc in the sense of Hausdorff (see, e.g., [2]) or inner and outer semicontinuity (see, e.g., [8], where it is shown that the last two concepts are equivalent to lsc in Berge sense and closedness when $\mathcal{M}(y)$ is closed for all $y \in Y)$.

2. Preliminaries. We say that $\mathcal{M}: Y \rightrightarrows \mathbb{R}^{n}$ is locally convex at $y_{0} \in Y$ if there exists an open set $V \subset Y$, containing $y_{0}$, such that $\mathcal{M}(y)$ is convex for all $y \in V$. We shall use the following sufficient condition for $\mathcal{M}$ to be locally bounded.

Proposition 2.1. Let $\mathcal{M}: Y \rightrightarrows \mathbb{R}^{n}$ and let $y_{0} \in \operatorname{dom} \mathcal{M}$ such that $\mathcal{M}\left(y_{0}\right)$ is bounded and $\mathcal{M}$ is lsc, closed, and locally convex at $y_{0}$. Then $\mathcal{M}$ is locally bounded and continuous at $y_{0}$.

Proof. Let $r_{0} \in \mathbb{N}$ such that

$$
\mathcal{M}\left(y_{0}\right) \subset B\left(0_{n} ; r_{0}\right) .
$$

Since $\mathcal{M}$ is lsc and locally convex at $y_{0}$ there exists an open set $V \subset Y$, containing $y_{0}$, such that $\mathcal{M}(y)$ is convex and

$$
B\left(0_{n} ; r_{0}\right) \cap \mathcal{M}(y) \neq \emptyset \text { for each } y \in V .
$$

If $\mathcal{M}$ is not locally bounded at $y_{0}$, given $r \in \mathbb{N}$ there exists $y_{r} \in Y$, with $\delta\left(y_{r}, y\right) \leq$ $\frac{1}{r}$, such that $\mathcal{M}\left(y_{r}\right) \nsubseteq B\left(0_{n} ; r\right)$. Thus there exists a sequence $\left\{x_{r}\right\}$ such that

$$
x_{r} \in \mathcal{M}\left(y_{r}\right),\left\|x_{r}\right\| \geq r, r=1,2, \ldots \text {. }
$$

Let $r_{1} \geq r_{0}$ such that $y_{r} \in V$ for all $r \geq r_{1}$. In this case, due to (2.2), we can take $z_{r} \in B\left(0_{n} ; r_{0}\right) \cap \mathcal{M}\left(y_{r}\right)$. Since $x_{r} \in \mathcal{M}\left(y_{r}\right) \backslash B\left(0_{n} ; r_{0}\right)$ and $\mathcal{M}\left(y_{r}\right)$ is convex, there exists $\left.\left.u_{r} \in\right] x_{r}, z_{r}\right]:=\left\{(1-\lambda) x_{r}+\lambda z_{r} \mid 0<\lambda \leq 1\right\}$ such that

$$
u_{r} \in \mathcal{M}\left(y_{r}\right),\left\|u_{r}\right\|=r_{0}, r \leq r_{1} .
$$

By the compactness of the spheres in $\mathbb{R}^{n}$, there exists a subsequence $\left\{u_{r_{k}}\right\}$ such that $u_{r_{k}} \in \mathcal{M}\left(y_{r_{k}}\right), k=1,2, \ldots$, and $\lim _{k} u_{r_{k}}=u_{0}$, with $\left\|u_{0}\right\|=r_{0}$. Since $\mathcal{M}$ is closed at $y_{0}$ and $\lim _{k} y_{r_{k}}=y_{0}$, we must have $u_{0} \in \mathcal{M}\left(y_{0}\right)$, which contradicts (2.1).

We have shown that $\mathcal{M}$ is locally bounded at $y_{0}$. Since we are assuming that $\mathcal{M}$ is closed at $y_{0}$, it is also usc at $y_{0}$. Hence it is continuous at $y_{0}$.

The condition of $\mathcal{M}$ being locally convex above is not superfluous as the following example shows.

Example 2.2. If $Y=[0,1]$ and $\mathcal{M}: Y \rightrightarrows \mathbb{R}$ is defined by $\mathcal{M}(y)=\{0,1 / y\}$ for $y \neq 0$ and $\mathcal{M}(0)=\{0\}$, then $\mathcal{M}$ is neither locally bounded nor continuous at $y_{0}=0$, in spite of $\mathcal{M}\left(y_{0}\right)$ being bounded and being $\mathcal{M}$ lsc and closed at $y_{0}$.

The truncated mapping of $\mathcal{M}: Y \rightrightarrows \mathbb{R}^{n}$ with radius $\rho>0$ is $\mathcal{M}_{\rho}: Y \rightrightarrows \mathbb{R}^{n}$ defined such as

$$
\mathcal{M}_{\rho}(y):=\mathcal{M}(y) \cap \operatorname{cl} B\left(0_{n} ; \rho\right) \text { for all } y \in Y .
$$

The following result (Lemma 2 in [5]), which establishes the relationships between $\mathcal{M}$ and $\mathcal{M}_{\rho}$, will be useful in the next sections.

Proposition 2.3. Let $\mathcal{M}: Y \rightrightarrows \mathbb{R}^{n}$ and let $y_{0} \in \operatorname{dom} \mathcal{M}$. Then the following 
statements hold:

(i) $\mathcal{M}$ is closed at $y_{0}$ if and only if $\mathcal{M}_{\rho}$ is closed at $y_{0}$ for all $\rho>0$ such that $\mathcal{M}_{\rho}\left(y_{0}\right) \neq \emptyset$.

(ii) If $\mathcal{M}$ is usc at $y_{0}$ and $\mathcal{M}\left(y_{0}\right)$ is closed, then $\mathcal{M}_{\rho}$ is usc at $y_{0}$ for all $\rho>0$ such that $\mathcal{M}_{\rho}\left(y_{0}\right) \neq \emptyset$.

(iii) If $\mathcal{M}$ is usc at $y_{0}$, then there exist a positive scalar $\bar{\rho}$ and an open neighborhood of $y_{0}, V$, such that

$$
\mathcal{M}(y) \backslash \mathcal{M}_{\bar{\rho}}(y) \subset \mathcal{M}\left(y_{0}\right) \backslash \mathcal{M}_{\bar{\rho}}\left(y_{0}\right) \quad \text { for all } y \in V .
$$

The converse statement holds when $\mathcal{M}$ is closed at $y_{0}$.

(iv) If $\mathcal{M}_{\rho}$ is lsc at $y_{0}$ for every $\rho$ such that $\mathcal{M}\left(y_{0}\right) \cap B\left(0_{n} ; \rho\right) \neq \emptyset$, then $\mathcal{M}$ is lsc at $y_{0}$. The converse statement holds if $\mathcal{M}\left(y_{0}\right)$ is convex.

As an immediate consequence of the following result we obtain characterizations of the identities $\mathcal{F}=\operatorname{conv} b d \mathcal{F}, \mathcal{F}=\operatorname{conv} \operatorname{rbd} \mathcal{F}$, and $\mathcal{F}=$ conv extr $\mathcal{F}$. Recall that an edge is a one-dimensional face whereas a half-flat is the intersection of a flat (also called affine manifold) with a closed halfspace which meets it, but does not contain it.

Proposition 2.4. Given a convex set $F \subset \mathbb{R}^{n}$, the following statements hold:

(i) $F=$ conv bd $F$ if and only if $F$ is a closed set which does not contain halfspaces.

(ii) $F=\operatorname{conv} \operatorname{rbd} F$ if and only if $F$ is a closed set which does not contain half-flats of the same dimension.

(iii) If $F=$ conv $\operatorname{extr} F$, then $F$ contains neither lines nor unbounded edges. The converse holds if $F$ is closed.

Proof. Obviously, if $F=\emptyset$, then

$$
\operatorname{conv} \operatorname{bd} F=\operatorname{conv} \operatorname{rbd} F=\operatorname{conv} \operatorname{extr} F=\emptyset .
$$

So we can assume that $F \neq \emptyset$ without loss of generality.

(i) It is a straightforward consequence of Lemma 2 in [3].

(ii) If $F=\operatorname{conv} \operatorname{rbd} F$, then $\operatorname{rbd} F \subset F$ and so $F$ is closed for each $y \in Y$. If $F$ contains a half-flat of the same dimension, then it is either a flat or a half-flat, with conv $\operatorname{rbd} F \neq F$ in both cases.

Conversely, since $F$ is a closed and convex set which is neither a flat nor a half-flat, then $F=\operatorname{conv} \operatorname{rbd} F$ by Theorem 2.6.12 in [9].

(iii) Suppose that $F=\operatorname{conv} \operatorname{extr} F . F \neq \emptyset$ entails $\operatorname{extr} F \neq \emptyset$ and so $F$ does not contain lines. We shall obtain a contradiction assuming the existence of a halfline edge of $F$, say $A$.

Let $A=\{\bar{x}+\lambda v \mid \lambda \geq 0\}$ be an edge of $F$. Then $v \neq 0_{n}$ and $\bar{x} \in \operatorname{extr} F$. We shall prove that no element of $A \backslash\{\bar{x}\}$ belongs to conv extr $F$. We assume the contrary, i.e., that there exists $\lambda>0$ such that $\bar{x}+\lambda v \in \operatorname{conv} \operatorname{extr} F$.

If $\bar{x}+\lambda v=x_{1} \in \operatorname{extr} F$, then $x_{1}=\frac{1}{2} \bar{x}+\frac{1}{2}(\bar{x}+2 \lambda v)$, with $\bar{x}, \bar{x}+2 \lambda v \in F$, making this impossible. Thus we can write $\bar{x}+\lambda v=\sum_{i=1}^{p} \lambda_{i} x_{i}$, where $p \geq 2, \sum_{i=1}^{p} \lambda_{i}=1$ and $\lambda_{i}>0$, and $x_{i} \in \operatorname{extr} F, i=1, \ldots, p$, with $x_{i} \neq x_{j}$ if $i \neq j$. Then we can write

$$
\bar{x}+\lambda v=\lambda_{1} x_{1}+\left(1-\lambda_{1}\right) \sum_{i=2}^{p}\left(\frac{\lambda_{i}}{1-\lambda_{1}}\right) x_{i},
$$

which yields $x_{1}, \sum_{i=2}^{p}\left(\frac{\lambda_{i}}{1-\lambda_{1}}\right) x_{i} \in A$ because $A$ is a face of $F$. Since $A \cap \operatorname{extr} F=\{\bar{x}\}$, $x_{1}=\bar{x}$, and so from (2.5) we get 


$$
\bar{x}+\frac{\lambda}{1-\lambda_{1}} v=\sum_{i=2}^{p}\left(\frac{\lambda_{i}}{1-\lambda_{1}}\right) x_{i}
$$

By taking into account again that $A$ is a face of $F$, we get the following contradiction: $x_{2}, \ldots, x_{p} \in A \cap \operatorname{extr} F=\{\bar{x}\}$.

We have shown that $(A \backslash\{\bar{x}\}) \cap \operatorname{conv} \operatorname{extr} F=\emptyset$. Since $\emptyset \neq A \backslash\{\bar{x}\} \subset F$, we conclude that conv extr $F \subsetneq F$.

Conversely, if $F$ is closed and does not contain lines, it is the convex hull of its extreme points and extreme directions (Corollary 2.6.15 in [9]). Since the assumption precludes the existence of extreme directions, we have conv extr $F=F$.

Remark 2.5. According to Proposition 2.4, if $\mathcal{F}=\operatorname{conv}$ bd $\mathcal{F}(\mathcal{F}=\operatorname{conv} \operatorname{rbd} \mathcal{F})$, then we have $\mathcal{F}_{\rho}=\operatorname{convbd} \mathcal{F}_{\rho}\left(\mathcal{F}_{\rho}=\operatorname{conv} \operatorname{rbd} \mathcal{F}_{\rho}\right.$, respectively) for all $\rho>0$. Nevertheless, in the case of $\mathcal{F}=$ conv extr $\mathcal{F}$, we need to show that $\mathcal{F}_{\rho}=\operatorname{conv} \operatorname{extr} \mathcal{F}_{\rho}$ because $\mathcal{F}$ could be not closed-valued. In order to do this, it is enough to prove that if $\mathcal{F}(y):=F=\operatorname{conv} \operatorname{extr} F$ and $x \in F_{\rho}$ with $\|x\|<\rho$, then $x \in \operatorname{conv} \operatorname{extr} F_{\rho}$. We can write

$$
x=\sum_{j \in J} \lambda_{j} x_{j},|J|<\infty, \sum_{j \in J} \lambda_{j}=1, \lambda_{j}>0 \text { and } x_{j} \in \operatorname{extr} F \text { for all } j \in J .
$$

Let $I=\left\{j \in J \mid\left\|x_{j}\right\|>\rho\right\}$. If $I=\emptyset$, then $x_{j} \in[\operatorname{extr} F]_{\rho} \subset \operatorname{extr} F_{\rho}$ for all $j \in J$ and so $x \in \operatorname{conv} \operatorname{extr} F_{\rho}$. Otherwise take an arbitrary $k \in I$. Let $x_{k}^{\prime} \in\left[x, x_{k}\right] \subset F$ such that $\left\|x_{k}^{\prime}\right\|=\rho$, so that $x_{k}^{\prime} \in \operatorname{extr} F_{\rho}$. If $x_{k}^{\prime}=(1-\mu) x+\mu x_{k}$, with $0<\mu<1$, and we denote $y_{j}=x_{j}$ for all $j \in J, j \neq k$, and $y_{k}=x_{k}^{\prime}$, we get an expression $x=\sum_{j \in J} \alpha_{j} y_{j}$, where $\sum_{j \in J} \alpha_{j}=1, \alpha_{j}>0$ and $y_{j} \in \operatorname{extr} F$ for all $j \in J$, but now the cardinality of the set $\left\{j \in J \mid\left\|y_{j}\right\|>\rho\right\}$ is $|I|-1$. After $|I|$ iterations of this procedure we get $x$ expressed as a convex combination of elements of extr $F_{\rho}$. In fact, if $\Phi$ is any operator that transforms convex sets in $\mathbb{R}^{n}$ into sets in $\mathbb{R}^{n}$ satisfying $[\Phi(\mathcal{F})]_{\rho} \subset \Phi\left(\mathcal{F}_{\rho}\right) \subset \mathcal{F}_{\rho}$ and $\{x \in \mathcal{F}(y) \mid\|x\|=\rho\} \subset \Phi\left(\mathcal{F}_{\rho}(y)\right)$ for all $y \in Y$, then

$$
\mathcal{F}=\operatorname{conv} \Phi(\mathcal{F}) \Longrightarrow \mathcal{F}_{\rho}=\operatorname{conv} \Phi\left(\mathcal{F}_{\rho}\right) .
$$

Observe that $\Phi(\mathcal{F})=$ bd $\mathcal{F}, \operatorname{rbd} \mathcal{F}$, and extr $\mathcal{F}$ satisfy these conditions.

3. Lower semicontinuity. We shall use the following classical result ([6, Proposition 2.6]).

Theorem 3.1. If $\mathcal{A}: Y \rightrightarrows \mathbb{R}^{n}$ is lsc at $y_{0} \in \operatorname{dom} \mathcal{A}$, then $\operatorname{conv} \mathcal{A}$ is also lsc at $y_{0}$.

In particular, taking $\mathcal{A}=$ bd $\mathcal{F}$ we get the direct statement of Proposition 1 in [5], whose corresponding converse statement establishes that, if $\mathcal{F}=$ conv bd $\mathcal{F}$ is lsc and closed at $y_{0} \in \operatorname{dom} \mathcal{F}$, then bd $\mathcal{F}$ is also lsc at $y_{0}$. The next two results are counterparts of this converse statement for $\operatorname{rbd} \mathcal{F}$ and extr $\mathcal{F}$ (instead of bd $\mathcal{F}$ ). Example 3 in [5], where bd $\mathcal{F}=\operatorname{rbd} \mathcal{F}=\operatorname{extr} \mathcal{F}$, shows that the closedness of $\mathcal{F}$ is not superfluous in these results. The following example shows that, in general, if $\operatorname{conv} \mathcal{A}$ is lsc and closed at $y_{0}$, then $\mathcal{A}$ is not necessarily lsc at $y_{0}$. Accordingly, the proofs must appeal to the specific properties of the sets $\operatorname{rbd} \mathcal{F}(y)$ and extr $\mathcal{F}(y)$.

Example 3.2. Let $\mathcal{A}: \mathbb{R} \rightrightarrows \mathbb{R}$ such that

$$
\mathcal{A}(y)=\left\{\begin{array}{cc}
\{-1,0,1\}, & y=0 \\
\{-1,1\}, & y \neq 0
\end{array}\right.
$$


It is easy to see that conv $\mathcal{A}$ is constant (so that it is continuous and closed) whereas $\mathcal{A}$ is not lsc at $y_{0}=0$.

TheOREM 3.3. Let $\mathcal{F}: Y \rightrightarrows \mathbb{R}^{n}$ be such that $\mathcal{F}=\operatorname{conv} \operatorname{rbd} \mathcal{F}$ and $\mathcal{F}$ is lsc and closed at $y_{0} \in \operatorname{dom} \mathcal{F}$. Then $\operatorname{rbd} \mathcal{F}$ is lsc at $y_{0}$.

Proof. Let us denote $\mathcal{R}=\operatorname{rbd} \mathcal{F}$. Since $\mathcal{F}\left(y_{0}\right)$ cannot be singleton (otherwise $\mathcal{R}\left(y_{0}\right)=\emptyset$, contradicting the assumptions), we have $\left|\mathcal{F}\left(y_{0}\right)\right|>1$.

We assume that $\mathcal{R}$ is not lsc at $y_{0}$ and we shall obtain a contradiction. This assumption entails the existence of an open convex set $W$ and a sequence $\left\{y_{r}\right\}$ such that $y_{r} \rightarrow y_{0}$,

$$
W \cap \mathcal{R}\left(y_{0}\right) \neq \emptyset
$$

and

$$
W \cap \mathcal{R}\left(y_{r}\right)=\emptyset, r=1,2, \ldots
$$

Since $y_{0} \in \operatorname{int} \operatorname{dom} \mathcal{F}$, we can assume that $y_{r} \in \operatorname{dom} \mathcal{F}, r=1,2, \ldots$ By $(3.1)$, we can choose a point $\widehat{x} \in W \cap \mathcal{R}\left(y_{0}\right)$. Fix $\bar{x} \in \operatorname{rint} \mathcal{F}\left(y_{0}\right)$. Then

$$
\widehat{x}-\lambda(\bar{x}-\widehat{x}) \notin \mathcal{F}\left(y_{0}\right) \text { for all } \lambda>0 .
$$

Because $\mathcal{F}$ is lsc at $y_{0}$ and $\bar{x}, \widehat{x} \in \mathcal{F}\left(y_{0}\right)$, there exist two sequences, $\left\{\bar{x}_{r}\right\}$ and $\left\{\widehat{x}_{r}\right\}$, with $\bar{x}_{r}, \widehat{x}_{r} \in \mathcal{F}\left(y_{r}\right)$ for all $r, \bar{x}_{r} \rightarrow \bar{x}$, and $\widehat{x}_{r} \rightarrow \widehat{x}$. Let $\delta>0$ such that $B(\widehat{x} ; \delta) \subset W$ and take $r_{0} \in \mathbb{N}$ such that $\widehat{x}_{r} \in B\left(\widehat{x} ; \frac{\delta}{2}\right)$ for all $r \geq r_{0}$. Given $r \geq r_{0}$, (3.2) yields $B\left(\widehat{x} ; \frac{\delta}{2}\right) \cap \mathcal{R}\left(y_{r}\right)=\emptyset$ and so, by $(1.1), B\left(\widehat{x} ; \frac{\delta}{2}\right) \cap$ aff $\mathcal{F}\left(y_{r}\right) \subset \mathcal{F}\left(y_{r}\right)$. Hence

$$
\widehat{x}_{r}-\frac{\delta}{4\left\|\bar{x}_{r}-\widehat{x}_{r}\right\|}\left(\bar{x}_{r}-\widehat{x}_{r}\right) \in \mathcal{F}\left(y_{r}\right) \quad \text { for all } r \geq r_{0} .
$$

Taking limits as $r \rightarrow \infty$ we get, by the closedness of $\mathcal{F}$ at $y_{0}$, that

$$
\widehat{x}-\frac{\delta}{4\|\bar{x}-\widehat{x}\|}(\bar{x}-\widehat{x}) \in \mathcal{F}\left(y_{0}\right),
$$

in contradiction with (3.3).

TheOREm 3.4. Let $\mathcal{F}: Y \rightrightarrows \mathbb{R}^{n}$ be such that $\mathcal{F}=$ conv extr $\mathcal{F}$ and $\mathcal{F}$ is lsc and closed at $y_{0} \in \operatorname{dom} \mathcal{F}$. Then $\operatorname{extr} \mathcal{F}$ is lsc at $y_{0}$.

Proof. We denote $\mathcal{E}=\operatorname{extr} \mathcal{F}$ and consider two possible cases.

Case 1. $\mathcal{F}\left(y_{0}\right)$ is bounded.

$\mathcal{F}$ is locally bounded at $y_{0}$ according to Proposition 2.1. Let $V$ be an open set in $Y, y_{0} \in V$, and $\rho>0$ such that $\mathcal{F}(y) \subset \operatorname{cl} B\left(0_{n} ; \rho\right)$ for all $y \in V$.

We assume that $\mathcal{E}$ is not lsc at $y_{0}$ and we shall get a contradiction.

Let $W$ be an open set and let $\left\{y_{r}\right\} \subset V$, with $y_{r} \rightarrow y_{0}$, be such that

$$
W \cap \mathcal{E}\left(y_{0}\right) \neq \emptyset
$$

and

$$
W \cap \mathcal{E}\left(y_{r}\right)=\emptyset \text { for all } r \in \mathbb{N} \text {. }
$$

By (3.4) we can select a point $x_{0} \in W \cap \mathcal{E}\left(y_{0}\right)$. 
Given $k \in \mathbb{N}$, since $x_{0} \in B\left(x_{0} ; k^{-1}\right) \cap \mathcal{F}\left(y_{0}\right)$ and $\mathcal{F}$ is lsc at $y_{0}$, there exists $r_{k} \in \mathbb{N}$ such that $B\left(x_{0} ; k^{-1}\right) \cap \mathcal{F}\left(y_{r_{k}}\right) \neq \emptyset$. We can assume that $\left\{y_{r_{k}}\right\}$ is a subsequence of $\left\{y_{r}\right\}$. Let

$$
z_{k} \in B\left(x_{0} ; k^{-1}\right) \cap \mathcal{F}\left(y_{r_{k}}\right), \quad k=1,2, \ldots
$$

For any $k \in \mathbb{N}$, we can write

$$
z_{k}=\sum_{i=1}^{n+1} \lambda_{i}^{k} e_{i}^{k}, \quad\left(\lambda_{1}^{k}, \ldots, \lambda_{n+1}^{k}\right) \in S, \quad e_{i}^{k} \in \mathcal{E}\left(y_{r_{k}}\right), \quad i=1, \ldots, n+1,
$$

because $\mathcal{F}\left(y_{r_{k}}\right)=\operatorname{conv} \mathcal{E}\left(y_{r_{k}}\right)$.

By the compactness of the simplex $S$, we can assume without loss of generality that $\left(\lambda_{1}^{k}, \ldots, \lambda_{n+1}^{k}\right) \rightarrow\left(\lambda_{1}, \ldots, \lambda_{n+1}\right) \in S$. Analogously, since for any $i \in\{1, \ldots$, $n+1\}$,

$$
\left\{e_{i}^{k}\right\} \subset \mathcal{E}\left(y_{r_{k}}\right) \subset \mathcal{F}\left(y_{r_{k}}\right) \subset \operatorname{cl} B\left(0_{n} ; \rho\right),
$$

we can assume that $e_{i}^{k} \rightarrow e_{i} \in \operatorname{cl} B\left(0_{n} ; \rho\right), i=1, \ldots, n+1$. Since $\mathcal{F}$ is closed at $y_{0}$ and $e_{i}^{k} \in \mathcal{F}\left(y_{r_{k}}\right)$ for all $k \in \mathbb{N}$, we get $e_{i} \in \mathcal{F}\left(y_{0}\right)$. Now, taking $\lim _{k}$ in (3.7) and recalling (3.6), we obtain

$$
x_{0}=\sum_{i=1}^{n+1} \lambda_{i} e_{i}, \quad\left(\lambda_{1}^{k}, \ldots, \lambda_{n+1}^{k}\right) \in S, \quad e_{i} \in \mathcal{F}\left(y_{0}\right), \quad i=1, \ldots, n+1 .
$$

Since $x_{0} \in \mathcal{E}\left(y_{0}\right)=\operatorname{extr} \mathcal{F}\left(y_{0}\right)$, we must have in (3.8) all the coefficients $\lambda_{i}=0$ except one, $\lambda_{j}=1$, in which case $x_{0}=e_{j}$. Since $e_{j}=\lim _{k} e_{j}^{k},\left\{e_{j}^{k}\right\} \subset \mathcal{E}\left(y_{r_{k}}\right) \subset \mathbb{R}^{n} \backslash W$ by (3.5), and $\mathbb{R}^{n} \backslash W$ is closed, we have $x_{0}=e_{j} \in \mathbb{R}^{n} \backslash W$, i.e., $x_{0} \notin W$. This contradicts the selection of $x_{0}$ in $W \cap \mathcal{E}\left(y_{0}\right)$.

Case 2. $\mathcal{F}\left(y_{0}\right)$ is unbounded.

The plan of the proof is to consider the truncated mapping $\mathcal{F}_{\rho}$, for a certain $\rho>0$. Since $\mathcal{F}_{\rho}=$ conv extr $\mathcal{F}_{\rho}$ by Remark 2.5 and $\mathcal{F}_{\rho}\left(y_{0}\right)$ is bounded, we are in case 1 and so extr $\mathcal{F}_{\rho}$ will be lsc at $y_{0}$. This will allow us to conclude that $\mathcal{E}=\operatorname{extr} \mathcal{F}$ is lsc at $y_{0}$.

First we show that if $\mathcal{E}_{\rho}$ is the truncated mapping of $\mathcal{E}$ of radius $\rho>0$, then

$$
\operatorname{extr} \mathcal{F}_{\rho}(y)=\mathcal{E}_{\rho}(y) \cup\{x \in \mathcal{F}(y) \mid\|x\|=\rho\} \text { for all } y \in Y \text {. }
$$

In fact, the inclusion extr $\mathcal{F}_{\rho}(y) \supset \mathcal{E}_{\rho}(y) \cup\{x \in \mathcal{F}(y) \mid\|x\|=\rho\}$ is obvious. For the reverse inclusion take $x \in \operatorname{extr} \mathcal{F}_{\rho}(y)$ such that $\|x\|<\rho$. Assume that $x=\lambda u+(1-\lambda) v$ with $0<\lambda<1$ and $u, v \in \mathcal{F}(y), u \neq v$. We may assume without loss of generality that $\|u\|,\|v\|<\rho$ which contradicts the fact that $x$ is an extreme point of $\mathcal{F}_{\rho}(y)$. Therefore, $x \in \mathcal{E}_{\rho}(y)$.

Now, in order to prove that $\mathcal{E}$ is lsc at $y_{0}$, assume that $\mathcal{E}$ is not. Then there exist $x_{0} \in \mathcal{E}\left(y_{0}\right), \delta>0$, and a sequence $\left\{y_{r}\right\}$ such that $y_{r} \rightarrow y_{0}$ and $\mathcal{E}\left(y_{r}\right) \cap B\left(x_{0} ; \delta\right)=\emptyset$ for every $r \in \mathbb{N}$. Take $\rho=\left\|x_{0}\right\|+\delta$ and observe that $x_{0} \in \operatorname{extr} \mathcal{F}_{\rho}\left(y_{0}\right)$ according to (3.9). $\mathcal{F}_{\rho}$ is lsc and closed at $y_{0}$, and so, by case 1 , extr $\mathcal{F}_{\rho}$ is lsc at $y_{0}$, which implies that there exists a sequence $\left\{x_{r}\right\}$ such that $x_{r} \rightarrow x_{0}, x_{r} \in \operatorname{extr} \mathcal{F}_{\rho}\left(y_{r}\right)$, and $\left\|x_{r}\right\|<\rho$ for $r$ large enough. This yields the contradiction $\mathcal{E}\left(y_{r}\right) \cap B\left(x_{0} ; \delta\right) \neq \emptyset$. 
4. Upper semicontinuity and closedness. In contrast with the lower semicontinuity, the closedness of a set-valued mapping $\mathcal{A}$ is not inherited by conv $\mathcal{A}$ (even though $\mathcal{A}=\operatorname{bd} \mathcal{F}, \operatorname{rbd} \mathcal{F}, \operatorname{extr} \mathcal{F}$, as Example 3 in [5] shows). On the other hand, Proposition 4 in [5] establishes that, if bd $\mathcal{F}$ is usc at $y_{0}$, then $\mathcal{F}$ is usc at $y_{0}$. In this section we shall prove that a similar statement holds for $\operatorname{rbd} \mathcal{F}$, but not for extr $\mathcal{F}$ even though extr $\mathcal{F}$ is either locally bounded or closed (nevertheless, according to the next Theorem 4.3, these two properties together entail the upper semicontinuity and the closedness of $\mathcal{F}$ ).

Example 4.1. Let $\mathcal{E}: Y \rightrightarrows \mathbb{R}^{2}$, where $Y=[2,+\infty[$ and

$$
\mathcal{E}(y)=\left\{x \in \mathbb{R}^{2} \mid\|x\|=1, x_{1}<y^{-1}\right\} \cup\{(y, 0)\} \text { for all } y \in Y .
$$

It is easy to see that $\mathcal{E}$ is locally bounded and continuous but not closed at $y_{0}=2$, and that it is the extreme points set mapping of $\mathcal{F}=\operatorname{conv} \mathcal{E}$. We shall prove that $\mathcal{F}$ is not usc at $y_{0}$. Let

$$
W:=\left\{x \in \mathbb{R}^{2}|\sqrt{3}| x_{2} \mid<2-x_{1}, x_{1}<2\right\} \cup B\left((2,0) ; \frac{1}{2}\right),
$$

$\mathcal{F}\left(y_{0}\right) \subset W$. If $y>2$, then $\bar{x}=\left(1, \frac{1}{\sqrt{3}}\right) \in \mathcal{F}(y) \backslash W$. Observe also that $\mathcal{F}$ cannot be closed at $y_{0}$ (because $\mathcal{F}\left(y_{0}\right)$ is not closed).

Example 4.2. Let $\mathcal{E}: \mathbb{R} \rightrightarrows \mathbb{R}^{3}$ be such that

$$
\mathcal{E}(y)=\left\{\left(x_{1}, x_{2}, 0\right) \in \mathbb{R}^{3} \mid x_{2}=x_{1}^{2}\right\} \cup\{(0,0, y)\} \text { for all } y \in \mathbb{R} .
$$

As in the previous example, $\mathcal{E}=\operatorname{extr} \mathcal{F}$ for $\mathcal{F}=\operatorname{conv} \mathcal{E}$ and $\mathcal{E}$ is continuous at $y_{0}=0$, but now $\mathcal{E}$ is also closed and $\mathcal{E}\left(y_{0}\right)$ is unbounded. In order to prove that $\mathcal{F}$ is not usc at $y_{0}$, let us consider the convex plane set $C:=\left\{x \in \mathbb{R}^{2} \mid x_{2} \geq x_{1}^{2}\right\}$ and the open set

$$
W:=\mathbb{R}^{3} \backslash\left\{x \in \mathbb{R}^{3} \mid x_{3} \geq x_{2}^{-1}, x_{2}>0\right\} .
$$

Obviously, $\mathcal{F}\left(y_{0}\right)=C \times\{0\} \subset W$. Moreover, if $y>0$ and $y>4 / r^{2}$ for $0 \neq r \in \mathbb{R}$, we have

$$
\left(0, \frac{r^{2}}{2}, \frac{y}{2}\right)=\frac{1}{2}(0,0, y)+\frac{1}{4}\left[\left(-r, r^{2}, 0\right)+\left(r, r^{2}, 0\right)\right] \in \mathcal{F}(y) \backslash W,
$$

so that $\mathcal{F}(y) \nsubseteq W$. Hence $\mathcal{F}$ is not usc at $y_{0}$.

Finally, we show that $\mathcal{F}$ is closed at $y_{0}$. Let $y_{r} \rightarrow y_{0}$ and $x^{r} \rightarrow x^{0}$ be such that $x^{r} \in \mathcal{F}\left(y_{r}\right), r=1,2, \ldots$. Since $\mathcal{F}\left(y_{r}\right)=\operatorname{conv}\left[(C \times\{0\}) \cup\left\{\left(0,0, y_{r}\right)\right\}\right]$, for any $r \in \mathbb{N}$, we can write

$$
x^{r}=\lambda_{r}\left(c^{r}, 0\right)+\left(1-\lambda_{r}\right)\left(0,0, y_{r}\right)=\left(\lambda_{r} c^{r},\left(1-\lambda_{r}\right) y_{r}\right), c^{r} \in C, 0 \leq \lambda_{r} \leq 1 .
$$

Observe that $c^{r} \in C$ and $(0,0) \in C$ entail $\lambda_{r} c^{r} \in C$. On the other hand, $x_{3}^{r}=$ $\left(1-\lambda_{r}\right) y_{r} \in \operatorname{conv}\left\{0, y_{r}\right\}$. Taking limits we get $x^{0}=\lim _{r} x^{r} \in C \times\{0\}=\mathcal{F}\left(y_{0}\right)$.

The next result is a reformulation of a well-known result ([1, Lemma 1.1.9]), taking into account the mentioned equivalence between closedness and outer semicontinuity.

TheOREm 4.3. If $\mathcal{A}: Y \rightrightarrows \mathbb{R}^{n}$ is closed and locally bounded at $y_{0} \in \operatorname{dom} \mathcal{A}$, then $\operatorname{conv} \mathcal{A}$ is closed and usc at $y_{0}$. 
Observe that it is not possible to replace in Theorem 4.3 above the condition " $\mathcal{A}$ is closed and locally bounded at $y_{0}$ " by just " $\mathcal{A}$ is closed and usc at $y_{0}$ " (recall Example 4.2).

Given two set-valued mappings $\mathcal{M}, \mathcal{N}: Y \rightrightarrows \mathbb{R}^{n}$, we say that $\mathcal{M}$ is contained in $\mathcal{N}$ (in brief, $\mathcal{M} \subset \mathcal{N}$ ) locally at $y_{0}$ if there exists an open set $V \subset Y$, containing $y_{0}$, such that $\mathcal{M}(y) \subset \mathcal{N}(y)$ for all $y \in V$. We also define the closure of $\mathcal{M}$ as the mapping $\operatorname{cl} \mathcal{M}: Y \rightrightarrows \mathbb{R}^{n}$ such that $(\operatorname{cl} \mathcal{M})(y)=\operatorname{cl} \mathcal{M}(y)$ for all $y \in Y$.

Corollary 4.4. Let $\mathcal{A}: Y \rightrightarrows \mathbb{R}^{n}$ and let $y_{0} \in \operatorname{dom} \mathcal{A}$ be such that $\mathcal{A}\left(y_{0}\right)$ is bounded and $\mathcal{A}$ is usc at $y_{0}$. Then each of the following conditions guarantees that $\operatorname{conv} \mathcal{A}$ is closed and usc at $y_{0}$ :

(i) $\mathcal{A}\left(y_{0}\right)$ is closed.

(ii) $\mathrm{cl} \mathcal{A} \subset \operatorname{conv} \mathcal{A}$ locally at $y_{0}$.

Proof. (i) Since $\mathcal{A}$ is usc at $y_{0}$ and $\mathcal{A}\left(y_{0}\right)$ is bounded, then $\mathcal{A}$ is locally bounded at $y_{0}$. The conclusion follows from Theorem 4.3.

(ii) First we prove that $\operatorname{cl} \mathcal{A}$ is usc at $y_{0}$. In fact, given an open set $W$ such that $\operatorname{cl} \mathcal{A}\left(y_{0}\right) \subset W$, we have

$$
\mathcal{A}\left(y_{0}\right) \subset U:=\operatorname{cl} \mathcal{A}\left(y_{0}\right)+B\left(0_{n} ; \varepsilon\right),
$$

where

$$
\varepsilon:=\frac{1}{2} d\left(\operatorname{cl} \mathcal{A}\left(y_{0}\right), \mathbb{R}^{n} \backslash W\right)>0 .
$$

Since $U$ is open, there exists an open set $V \subset Y, y_{0} \in V$, such that $\mathcal{A}(y) \subset U$ for all $y \in V$. Then $\operatorname{cl} \mathcal{A}(y) \subset \operatorname{cl} U \subset W$.

Now we show that conv $\mathcal{A}$ is usc at $y_{0}$.

Since $\operatorname{cl} \mathcal{A}$ is usc at $y_{0}$ and $\operatorname{cl} \mathcal{A}\left(y_{0}\right)$ is compact we can assert, applying statement (i) to $\operatorname{cl} \mathcal{A}$, that $\operatorname{conv} \operatorname{cl} \mathcal{A}$ is closed and usc at $y_{0}$. Since the assumption implies that conv $\operatorname{cl} \mathcal{A}=\operatorname{conv} \mathcal{A}$ locally at $y_{0}$, we conclude that $\operatorname{conv} \mathcal{A}$ is closed and usc at $y_{0}$.

The boundedness assumption in Corollary 4.4 is not superfluous even for the extreme points set mapping (recall again Example 4.2, where (i) holds).

Now, we give a condition that assures that if $\mathcal{A}$ is usc at $y_{0}$, then $\operatorname{conv} \mathcal{A}$ is usc at $y_{0}$ as well.

Proposition 4.5. Let $\mathcal{A}: Y \rightrightarrows \mathbb{R}^{n}$ and let $y_{0} \in \operatorname{dom} \mathcal{A}$ be such that

$$
\operatorname{rbd} \operatorname{conv} \mathcal{A} \subset \mathcal{A} \subset \operatorname{conv} \operatorname{rbd} \operatorname{conv} \mathcal{A}
$$

locally at $y_{0}$ and $\operatorname{conv} \mathcal{A}$ is closed at $y_{0}$. If $\mathcal{A}$ is usc at $y_{0}$, then $\operatorname{conv} \mathcal{A}$ is usc at $y_{0}$.

Proof. Let $\mathcal{F}:=\operatorname{conv} \mathcal{A}$ and let $\mathcal{R}=\operatorname{rbd} \mathcal{F}$. We assume that $\mathcal{A}$ is usc at $y_{0}$.

Let $V_{1}$ be a neighborhood of $y_{0}$ such that $\mathcal{R}(y) \subset \mathcal{A}(y) \subset \operatorname{conv} \mathcal{R}(y)$ for all $y \in V_{1}$. Then we have $\mathcal{F}(y)=\operatorname{conv} \mathcal{R}(y)$ for all $y \in V_{1}$.

By Proposition 2.3, there exists $\bar{\rho}>0$ and a neighborhood of $y_{0}, V_{2} \subset V_{1}$, such that

$$
\mathcal{A}(y) \backslash \mathcal{A}_{\bar{\rho}}(y) \subset \mathcal{A}\left(y_{0}\right) \backslash \mathcal{A}_{\bar{\rho}}\left(y_{0}\right) \text { for all } y \in V_{2} .
$$

We shall prove that we can replace $\mathcal{A}$ with $\mathcal{F}$ in (4.1), so that $\mathcal{F}$ will be usc at $y_{0}$ because $\mathcal{F}$ is closed at $y_{0}$ (again by Proposition 2.3). Let $\bar{y} \in V_{2}$ and $\bar{x}$ be such that

$$
\bar{x} \in \mathcal{F}(\bar{y}) \text { and }\|\bar{x}\|>\bar{\rho} .
$$


If $\bar{x} \in \mathcal{A}(\bar{y})$, then $\bar{x} \in \mathcal{A}(\bar{y}) \backslash \mathcal{A}_{\bar{\rho}}(\bar{y})$ and so

$$
\bar{x} \in \mathcal{A}\left(y_{0}\right) \backslash \mathcal{A}_{\bar{\rho}}\left(y_{0}\right) \subset \mathcal{A}\left(y_{0}\right) \subset \mathcal{F}\left(y_{0}\right) .
$$

Suppose that $\bar{x} \notin \mathcal{A}(\bar{y})$ and $\bar{x} \notin \mathcal{F}\left(y_{0}\right)$. Now, $\mathcal{R}(\bar{y}) \subset \mathcal{A}(\bar{y})$ implies that

$$
\bar{x} \in \mathcal{F}(\bar{y}) \backslash \mathcal{A}(\bar{y}) \subset \mathcal{F}(\bar{y}) \backslash \mathcal{R}(\bar{y})=\operatorname{rint} \mathcal{F}(\bar{y}) .
$$

Since $\mathcal{F}\left(y_{0}\right)$ is closed and convex, there exist $a \neq 0_{n}$ and a scalar $\alpha$ such that

$$
a^{\prime} \bar{x}=\alpha \text { and } a^{\prime} x<\alpha \text { for all } x \in \mathcal{F}\left(y_{0}\right) .
$$

Consider the flat $H:=\left\{x \in\right.$ aff $\left.\mathcal{F}(\bar{y}) \mid a^{\prime} x=\alpha\right\}$. Obviously $a^{\prime} c=0$ for all $c \in$ $H-\bar{x}$ (the linear subspace parallel to $H$ ).

We shall get a contradiction if we are able to prove that $H \subset \mathcal{F}(\bar{y})$. In fact, in this case if $a^{\prime} x=\alpha$ for all $x \in$ aff $\mathcal{F}(\bar{y})$, then $H=\operatorname{aff} \mathcal{F}(\bar{y})$ and so $\mathcal{F}(\bar{y})=$ aff $\mathcal{F}(\bar{y})$, i.e., $\mathcal{F}(\bar{y})$ is a flat. Otherwise $\mathcal{F}(\bar{y})$ is a half-flat. In both cases $\mathcal{F}(\bar{y}) \neq \operatorname{conv} \mathcal{R}(\bar{y})$ despite of $\bar{y} \in V_{1}$.

In order to prove that $H \subset \mathcal{F}(\bar{y})$ we associate with each $c \in(H-\bar{x}) \backslash\left\{0_{n}\right\}$ the halfline $S(c):=\{\bar{x}+\lambda c \mid \lambda \geq 0\} \subset H$. Now we prove that

$$
S(c) \cap \operatorname{cl} B\left(0_{n} ; \bar{\rho}\right)=\emptyset \Rightarrow S(c) \subset \operatorname{rint} \mathcal{F}(\bar{y}) .
$$

Assume that $S(c) \cap \operatorname{cl} B\left(0_{n} ; \bar{\rho}\right)=\emptyset$ and $S(c) \nsubseteq \operatorname{rint} \mathcal{F}(\bar{y})$. By (4.2) we have

$$
0<\bar{\lambda}:=\sup \left\{\lambda \in \mathbb{R}_{+} \mid \bar{x}+\lambda c \in \operatorname{rint} \mathcal{F}(\bar{y})\right\}<+\infty .
$$

Thus $\bar{x}+\bar{\lambda} c \in \mathcal{R}(\bar{y}) \subset \mathcal{A}(\bar{y})$ and, by (4.1), we have

$$
\begin{gathered}
\bar{x}+\bar{\lambda} c \in \mathcal{A}(\bar{y}) \backslash \operatorname{cl} B\left(0_{n} ; \bar{\rho}\right)=\mathcal{A}(\bar{y}) \backslash \mathcal{A}_{\bar{\rho}}(\bar{y}) \\
\subset \mathcal{A}\left(y_{0}\right) \backslash \mathcal{A}_{\bar{\rho}}\left(y_{0}\right) \subset \mathcal{F}\left(y_{0}\right),
\end{gathered}
$$

so that by (4.3) $a^{\prime} \bar{x}=\alpha$ and $a^{\prime} \bar{x}=a^{\prime}(\bar{x}+\bar{\lambda} c)<\alpha$. This is a contradiction.

Finally, we prove that $H \subset \mathcal{F}(\bar{y})$ by means of a discussion based on the set $C:=H \cap \operatorname{cl} B\left(0_{n} ; \bar{\rho}\right)$.

If $C=\emptyset$, then $H$ is the union of halflines emanating from $\bar{x}$ in all directions parallel to $H$, and these halflines are contained in $\operatorname{rint} \mathcal{F}(\bar{y})$, according to (4.4). Then $H \subset \operatorname{rint} \mathcal{F}(\bar{y}) \subset \mathcal{F}(\bar{y})$.

If $|C|=1$, then all the halflines mentioned above are contained in $\operatorname{rint} \mathcal{F}(\bar{y})$, except one. Thus $H \subset \mathcal{F}(\bar{y})$.

If $|C|>1$, then $C$ is a closed ball in $H$ and all the halflines in $H$ emanating from $\bar{x}$ which do not meet $C$ are contained in $\operatorname{rint} \mathcal{F}(\bar{y})$. Then $\mathcal{F}(\bar{y})$ contains the complement, relative to $H$, of a pointed cone with apex $\bar{x}$. Hence we have again $H \subset \mathcal{F}(\bar{y})$.

Given $\mathcal{A}: Y \rightrightarrows \mathbb{R}^{n}$ and $\rho>0$, we denote by $\mathcal{A}_{\rho}$ and by $(\operatorname{conv} \mathcal{A})_{\rho}$ the truncated mappings of $\mathcal{A}$ and conv $\mathcal{A}$, respectively, with radius $\rho$. We also define the mapping $\mathcal{A}^{\rho}: Y \rightrightarrows \mathbb{R}^{n}$ such that

$$
\mathcal{A}^{\rho}(y)=\mathcal{A}_{\rho}(y) \cup\{x \in \operatorname{conv} \mathcal{A}(y) \mid\|x\|=\rho\} .
$$

If $\mathcal{F}=\operatorname{conv} \operatorname{rbd} \mathcal{F}(\mathcal{F}=\operatorname{conv} b d \mathcal{F})$, and $\mathcal{A}=\operatorname{rbd} \mathcal{F}(\mathcal{A}=\operatorname{bd} \mathcal{F}$, respectively $)$, then $(\operatorname{conv} \mathcal{A})_{\rho}=\operatorname{conv} \mathcal{A}^{\rho}$. The inclusion $(\operatorname{conv} \mathcal{A})_{\rho} \subset \operatorname{conv} \mathcal{A}^{\rho}$ follows from the fact that any convex combination $x=(1-\lambda) u+\lambda v, 0 \leq \lambda \leq 1, x, u, v \in \operatorname{conv} \mathcal{A}(y)$, 
$\|x\| \leq \rho$ and $\|v\|>\rho$, can be expressed as $x=(1-\alpha) u+\alpha w$, where $0 \leq \alpha \leq 1$ and $w \in[x, v] \subset \operatorname{conv} \mathcal{A}(y)$, with $\|w\|=\rho$.

Lemma 4.6. Let $\mathcal{A}: Y \rightrightarrows \mathbb{R}^{n}$ and let $y_{0} \in \operatorname{dom} \mathcal{A}$ be such that $\mathcal{A}\left(y_{0}\right)$ and $\operatorname{conv} \mathcal{A}\left(y_{0}\right)$ are closed and $\mathcal{A}$ is usc at $y_{0}$. Then $\left\{\rho>0 \mid \mathcal{A}^{\rho}\right.$ is closed at $\left.y_{0}\right\}$ is unbounded.

Proof. We will prove that, under the assumptions, $\mathcal{A}^{\rho}$ is closed at $y_{0}$ for all $\rho \in I:=\left\{\rho>0 \mid \mathcal{A}_{\rho}\left(y_{0}\right) \neq \emptyset\right\}$ ( $I$ is a halfline). We denote $\mathcal{F}=\operatorname{conv} \mathcal{A}$.

Let $\rho \in I, y_{k} \rightarrow y_{0}$ and $x_{k} \rightarrow x_{0}$ be such that $x_{k} \in \mathcal{A}^{\rho}\left(y_{k}\right), k=1,2, \ldots$.

Since $\mathcal{A}^{\rho}\left(y_{k}\right) \subset \operatorname{cl} B\left(0_{n} ; \rho\right)$ for all $k \in \mathbb{N},\left\|x_{0}\right\| \leq \rho$.

If there exists an increasing sequence $\left\{k_{r}\right\} \subset \mathbb{N}$ such that $x_{k_{r}} \in \mathcal{A}\left(y_{k_{r}}\right), r=$ $1,2, \ldots$, then $x_{0} \in \mathcal{A}\left(y_{0}\right)$ (because $\mathcal{A}$ is closed at $\left.y_{0}\right)$ and so $x_{0} \in \mathcal{A}_{\rho}\left(y_{0}\right) \subset \mathcal{A}^{\rho}\left(y_{0}\right)$.

Thus we can assume without loss of generality that $x_{k} \notin \mathcal{A}\left(y_{k}\right), k=1,2, \ldots$.

Given $k \in \mathbb{N}$, we have $x_{k} \in \mathcal{A}^{\rho}\left(y_{k}\right) \backslash \mathcal{A}_{\rho}\left(y_{k}\right) \subset\left\{x \in \mathcal{F}\left(y_{k}\right) \mid\|x\|=\rho\right\}$. Since $\left\|x_{k}\right\|=\rho$ for all $k$, we have $\left\|x_{0}\right\|=\rho$.

If $x_{0} \in \mathcal{F}\left(y_{0}\right)$, then $x_{0} \in \mathcal{A}^{\rho}\left(y_{0}\right)$ and we have finished. So we assume that $x_{0} \notin \mathcal{F}\left(y_{0}\right)$. Since this set is closed, $\varepsilon:=\frac{1}{2} d\left(x_{0}, \mathcal{F}\left(y_{0}\right)\right)>0$. Let us consider the open convex set $W:=\mathcal{F}\left(y_{0}\right)+B\left(0_{n} ; \varepsilon\right)$. Since $\mathcal{A}\left(y_{0}\right) \subset \mathcal{F}\left(y_{0}\right) \subset W$ and $\mathcal{A}$ is usc at $y_{0}$, there exists a neighborhood of $y_{0}$, say $V$, such that $\mathcal{A}(y) \subset W$ for all $y \in V$. Then, taking convex hulls, we get $\mathcal{F}(y) \subset W$ for all $y \in V$.

Let $k_{0} \in \mathbb{N}$ be such that $y_{k} \in V$ for all $k \geq k_{0}$. For such a $k$ we have $x_{k} \in$ $\mathcal{A}^{\rho}\left(y_{k}\right) \subset \mathcal{F}\left(y_{k}\right) \subset W$ whereas $x_{0} \notin \mathcal{F}\left(y_{0}\right)$, so that $d\left(x_{k}, x_{0}\right) \geq \varepsilon$. This contradicts $x_{k} \rightarrow x_{0}$.

LEMma 4.7. Let $\mathcal{A}: Y \rightrightarrows \mathbb{R}^{n}$ be such that $(\operatorname{conv} \mathcal{A})_{\rho}=\operatorname{conv} \mathcal{A}^{\rho}$ for all $\rho>0$ sufficiently large and let $y_{0} \in \operatorname{dom} \mathcal{A}$ such that $\left\{\rho>0 \mid \mathcal{A}^{\rho}\right.$ is closed at $\left.y_{0}\right\}$ is unbounded. Then conv $\mathcal{A}$ is closed at $y_{0}$.

Proof. Let $\mathcal{F}:=\operatorname{conv} \mathcal{A}$. Let $y_{r} \rightarrow y_{0}$ and $x_{r} \rightarrow x_{0}$ be such that $x_{r} \in \mathcal{F}\left(y_{r}\right)$, $r=1,2, \ldots$.

Since the convergent sequence $\left\{x_{r}\right\}$ is bounded, and by the assumptions on $\left\{\mathcal{A}^{\rho} \mid \rho>0\right\}$, there exists $\rho>0$ such that $\left\|x_{r}\right\| \leq \rho$ for all $r \in \mathbb{N}, \mathcal{F}_{\rho}=\operatorname{conv} \mathcal{A}^{\rho}$ and $\mathcal{A}^{\rho}$ is closed at $y_{0}$. Since $\mathcal{A}^{\rho}$ is closed and locally bounded at $y_{0}$, by Theorem 4.3, $\mathcal{F}_{\rho}=\operatorname{conv} \mathcal{A}^{\rho}$ is closed and usc at $y_{0}$. Then, since $x_{r} \in \mathcal{F}_{\rho}\left(y_{r}\right)$ for all $r \in \mathbb{N}$, we have $x_{0} \in \mathcal{F}_{\rho}\left(y_{0}\right) \subset \mathcal{F}\left(y_{0}\right)$.

Proposition 4.8. Let $\mathcal{A}: Y \rightrightarrows \mathbb{R}^{n}$ be such that $(\operatorname{conv} \mathcal{A})_{\rho}=\operatorname{conv} \mathcal{A}^{\rho}$ for all $\rho>0$ sufficiently large and let $y_{0} \in \operatorname{dom} \mathcal{A}$ such that $\mathcal{A}\left(y_{0}\right)$ is closed,

$$
\operatorname{rbd} \operatorname{conv} \mathcal{A} \subset \mathcal{A} \subset \operatorname{conv} \operatorname{rbd} \operatorname{conv} \mathcal{A}
$$

locally at $y_{0}$ and $\mathcal{A}$ is usc at $y_{0}$. Then conv $\mathcal{A}$ is usc at $y_{0}$.

Proof. By assumption rbd conv $\mathcal{A}\left(y_{0}\right) \subset \mathcal{A}\left(y_{0}\right) \subset \operatorname{conv} \mathcal{A}\left(y_{0}\right)$, so that $\operatorname{conv} \mathcal{A}\left(y_{0}\right)$ is closed. Then, by Lemma 4.6, $\left\{\rho>0 \mid \mathcal{A}^{\rho}\right.$ is closed at $\left.y_{0}\right\}$ is unbounded and, by Lemma 4.7, $\operatorname{conv} \mathcal{A}$ is closed at $y_{0}$. We conclude that $\operatorname{conv} \mathcal{A}$ is usc at $y_{0}$ by Proposition 4.5 .

TheOREM 4.9. Let $\mathcal{F}: Y \rightrightarrows \mathbb{R}^{n}$ be such that $\mathcal{F}=\operatorname{conv} \operatorname{rbd} \mathcal{F}$ and $\operatorname{rbd} \mathcal{F}$ is usc at $y_{0} \in \operatorname{dom} \mathcal{F}$. Then $\mathcal{F}$ is usc at $y_{0}$.

Proof. It is a straightforward consequence of Proposition 4.8, taking $\mathcal{A}=\operatorname{rbd}$ $\mathcal{F}$.

The last four results are also valid replacing "rbd" everywhere with "bd" (see [5]). The final example illustrates the results in sections 3 and 4 and shows that there is no usc counterpart for Theorems 3.3 and 3.4. 
Example 4.10. Let us identify the complex field $\mathbb{C}$ with $\mathbb{R}^{2}$ and let us take as $Y$ the set of polynomials of degree $q \in \mathbb{N}$ (fixed) with complex coefficients equipped with the Euclidean distance on $\mathbb{R}^{2 q+2}$. Given $y \in Y$, we denote by $\mathcal{A}(y)$ its set of complex zeros and by $\mathcal{F}(y)$ its convex hull, i.e., the polytope $\mathcal{F}(y)=\operatorname{conv} \mathcal{A}(y)$. By the fundamental theorem of algebra, $\mathcal{A}(y) \neq \emptyset$ for all $y \in Y$, so that $\operatorname{dom} \mathcal{A}=Y$. Let us denote by $\mathcal{B}, \mathcal{R}$, and $\mathcal{E}$ the boundary mapping, the relative boundary mapping, and the extreme points set mapping of $\mathcal{F}$, respectively. By Proposition 2.4, we have

$$
\mathcal{F}=\operatorname{conv} \mathcal{B}=\operatorname{conv} \mathcal{R}=\operatorname{conv} \mathcal{E} .
$$

$\mathcal{A}$ is lsc and usc as a consequence of a well-known consequence of Rolle's theorem for complex polynomials (see, e.g., [7]) and, since it has closed images, it is also closed. By Theorem 3.1 and Corollary $4.4, \mathcal{F}$ is also lsc, usc, and closed. Consequently, $\mathcal{B}$, $\mathcal{R}$, and $\mathcal{E}$ are lsc by Propositions 1 in [4] and Theorems 3.3 and 3.4 in this paper (the direct proofs of these statements are rather involved). Now we show that $\mathcal{R}$ and $\mathcal{E}$ are neither usc nor closed if $q=3$.

Let $y_{0}=x^{3}+x$, with $\mathcal{A}\left(y_{0}\right)=\{0, \pm i\}$, and let $y_{r}=x^{3}-\frac{2}{r} x^{2}+\left(1+\frac{1}{r^{2}}\right) x$, with $\mathcal{A}\left(y_{r}\right)=\left\{0, \frac{1}{r} \pm i\right\}, r=1,2, \ldots$. Obviously, $y_{r} \rightarrow y_{0}$. Taking the constant sequence $x_{r}=0, r=1,2, \ldots$, we have $x_{r} \in \mathcal{E}\left(y_{r}\right) \subset \mathcal{F}\left(y_{r}\right)$ for all $r$, whereas $0 \notin \mathcal{E}\left(y_{0}\right)=$ $\mathcal{R}\left(y_{0}\right)=\{ \pm i\}$. Thus neither $\mathcal{R}$ nor $\mathcal{E}$ is closed (usc) at $y_{0}$.

Acknowledgment. The authors wish to thank the referees for their valuable comments and suggestions.

\section{REFERENCES}

[1] J.-P. Aubin and H. Frankowska, Set-Valued Analysis, Birkhäuser, Boston, 1990.

[2] B. Bank, J. Guddat, D. Klatte, B. Kummer, and K. Tammer, Nonlinear Parametric Optimization, Birkhäuser Verlag, Basel, Switzerland, 1983.

[3] M. A. Goberna, M. Larriqueta, and V. N. Vera de Serio, On the stability of the boundary of the feasible set in linear optimization, Set-Valued Anal., 11 (2003), pp. 203-223.

[4] M. A. Goberna, M. Larriqueta, and V. N. Vera de Serio, On the stability of the extreme point set in linear optimization, SIAM J. Optim., 15 (2005), pp. 1155-1169.

[5] M. A. Goberna, M. A. López, And M. I. Todorov, On the stability of closed-convex-valued mappings and the associated boundaries, J. Math. Anal. Appl., 306 (2005), pp. 502-515.

[6] E. Michael, Continuous selections. I., Ann. of Math., 63 (1956), pp. 361-382.

[7] J. E. Marsden and M. J. Hoffman, Basic Complex Analysis, W. H. Freeman, New York, 1987.

[8] R. Rockafellar and R. B. Wets, Variational Analysis, Springer-Verlag, Berlin, 1998.

[9] R. Webster, Convexity, Oxford University Press, Oxford, 1994. 